\title{
Precursor experiments to search for permanent electric dipole moments (EDMs) of protons and deuterons at COSY
}

\author{
Frank Rathmann* \\ Institut für Kernphysik and Jülich Center for Hadron Physics, Forschungszentrum Jülich, 52425 \\ Jülich, Germany \\ E-mail: f.rathmannefz-juelich.de
}

\section{Nikolai Nikolaev}

Institut für Kernphysik and Jülich Center for Hadron Physics, Forschungszentrum Jülich, 52425

Jülich, Germany, and Landau Institute, 142432 Chernogolovka, Russia

E-mail: h.nikolaevefz-juelich.de

In this presentation we discuss a number of experiments on the search for proton or deuteron EDMs, which could be carried out at COSY-Jülich. Most promising is the use of an radiofrequency radial electric field flipper that would lead to the accumulation of a $C P$ violating inplane beam polarization. Most crucial for storage ring searches for EDMs is the spin-coherence time, and we report on analytic evaluations which point at a much larger spin-coherence time for deuterons by about a factor of 200 compared to the one for protons, and at COSY, the spin coherence time for deuterons could amount to about $10^{5} \mathrm{~s}$.

8th International Conference on Nuclear Physics at Storage Rings-Stori11,

October 9-14, 2011

INFN, Laboratori Nazionali di Frascati, Italy

\footnotetext{
* Speaker.
} 


\section{Introduction}

Electric dipole moments (EDM) are one of the keys to understand the origin of our Universe. The Universe as we know it has a microscopic net baryon number - about 0.2 baryons per cubic meter, or $\sim 10^{-10}$ of the density of relic photons. In 1967 Andrei Sakharov formulated three conditions for baryogenesis [四:

1. Early in the evolution of the universe, the baryon number conservation must be violated sufficiently strongly,

2. the $C$ and $C P$ invariances, and $T$ invariance thereof, must be violated, and

3. at the moment when the baryon number is generated, the evolution of the universe must be out of thermal equilibrium.

$C P$ violation in kaon decays is known since 1964 , it has been observed in $B$-decays and charmed meson decays. The Standard Model (SM) accommodates $C P$ violation via the phase in the Cabibbo-Kobayashi-Maskawa matrix. $C P$ and $P$ violation entail non-vanishing $P$ and $T$ violating electric dipole moments (EDMs) of elementary particles. Although extremely successful in many aspects, the SM has at least two weaknesses: neutrino oscillations do require extensions of SM and, most importantly, the SM mechanisms fails miserably in the expected baryogenesis rate. Simultaneously, the SM predicts an exceedingly small electric dipole moment of nucleons $10^{-33}<d_{n}<10^{-31} \mathrm{e} \cdot \mathrm{cm}$, way below the current upper bound for the neutron EDM, $d_{n}<2.9 \times 10^{-26} \mathrm{e} \cdot \mathrm{cm}$, and also beyond the reach of future EDM searches [ [ $]$ ]. In the quest for physics beyond the SM one could follow either the high energy trail or look into new methods which offer very high precision and sensitivity. Supersymmetry is one of the most attractive extensions of the SM and S. Weinberg emphasized in 1992 [B]]: "Endemic in supersymmetric (SUSY) theories are $C P$ violations that go beyond the SM. For this reason it may be that the next exciting thing to come along will be the discovery of a neutron electric dipole moment." The SUSY predictions span typically $10^{-29}<d_{n}<10^{-24} \mathrm{e} \cdot \mathrm{cm}$ and precisely this range is targeted in the new generation of EDM searches []].

There is consensus among theorists that measuring the EDM of the proton, deuteron and helion is as important as that of the neutron. Furthermore, it has been argued some 25 years ago that $T$ violating nuclear forces could substantially enhance nuclear EDMs [ [ 9 , 国]. At the moment, there are no significant direct upper bounds available on $d_{p}$ or $d_{d}$.

Non-vanishing EDMs give rise to a precession of the spin of a particle in an electric field. While ultra-cold electrically neutral atoms and neutrons can conveniently by stored in traps, the EDM of charged particle can only be approached with storage rings. An ambitious quest for a measurement of the EDM of the proton with envisioned sensitivity down to $d_{p} \sim 10^{-29} \mathrm{e} \cdot \mathrm{cm}$ is under development at BNL [ 6$]$. The principal idea is to store protons with longitudinal polarization in a purely electrostatic ring: the EDM would cause a precession around the radial electric field and thus lead to a build-up of transverse polarization which could be measured by standard polarimetry. Related ideas on dedicated storage rings for the deuteron and helion EDM are being discussed at IKP of Forschungszentrum Jülich within the newly found JEDI collaboration ${ }^{1}$.

\footnotetext{
${ }^{1}$ Jülich Electric Dipole moment Investigations
} 
Before jumping into construction of dedicated storage rings, it is imperative to test technical issues at existing facilities. Here we review several ideas for precursor experiments which could be performed at COSY, and which are subject to very modest additions or modifications to the existing machine. In a magnetic ring like COSY, the stable polarization axis in the absence of longitudinal magnetic fields, is normal to the ring plane, and at the heart of the most promising proposal is a radio-frequency electric field (RFE) spin flipper which would rotate the spin into the ring plane. The RFE flipper enables a coherent build-up of the EDM-generated $P$ and $T$ non-invariant in-plane polarization which can be determined from the up-down asymmetry of the scattering of stored particles on the polarimeter. Unless show stoppers pop up, one could theoretically aim for an upper bound for the deuteron of $d_{d}<10^{-24} \mathrm{e} \cdot \mathrm{cm}$, which would be as valuable as the existing upper bounds on $d_{n}$ [䧃, 回].

\section{EDM searches: state of the art}

The question of whether particles possess permanent electric dipole moments has a longstanding history, starting from the first measurement by Smith, Purcell, and Ramsey [四] for a neutron EDM as a signature for parity $(P)$ and time-reversal ( $T$ or $C P$ ) violation, which, over the last 50 years or so, resulted in ever decreasing upper limits. In Table $\mathbb{W}$, we give current and anticipated EDM bounds and sensitivities for nucleons, atoms, and the deuteron. The second-to-last column provides a rough measure of their probing power relative to the neutron $\left(d_{n}\right)$. At this level, storage ring EDM measurements bear the potential of an order of magnitude higher sensitivity than the currently planned neutron EDM experiments at SNS (Oak Ridge), ILL (Grenoble-France), and PSI (Villigen, Switzerland) [8]].

Table 1: Current EDM limits in units of [e.cm], and long-term goals for the neutron, ${ }^{199} \mathrm{Hg},{ }^{129} \mathrm{Xe}$, proton, and deuteron are given here. Neutron equivalent values indicate the EDM value for the neutron to provide the same physics reach as the indicated system.

\begin{tabular}{|c|c|c|c|c|}
\hline Particle & Current Limit & Goal & $d_{n}$ equivalent & reference \\
\hline Neutron & $<2.9 \times 10^{-26}$ & $\approx 10^{-28}$ & $10^{-28}$ & [Q] \\
\hline${ }^{199} \mathrm{Hg}$ & $<3.1 \times 10^{-29}$ & $10^{-29}$ & $10^{-26}$ & [ए]] \\
\hline${ }^{129} \mathrm{Xe}$ & $<6.0 \times 10^{-27}$ & $\approx 10^{-30}-10^{-33}$ & $\approx 10^{-26}-10^{-29}$ & [ㅁ] ] \\
\hline Proton & $<7.9 \times 10^{-25}$ & $\approx 10^{-29}$ & $10^{-29}$ & [ए0] \\
\hline Deuteron & & $\approx 10^{-29}$ & $3 \times 10^{-29}-5 \times 10^{-31}$ & \\
\hline
\end{tabular}

\section{Search for electric dipole moments of protons, deuterons, and ${ }^{3} \mathrm{He}$ at COSY}

EDM searches of charged fundamental particles have hitherto been impossible, because of the absence of the required new class of electrostatic storage rings. The new idea pursued at BNL is to freeze the horizontal spin motion, i.e., forcing the particles' spin to always point along the direction of motion, which cancels the $(g-2)$ precession. During the spin coherence time, the build-up of 
a vertical polarization component in the beam would indicate the signal for a finite EDM of the orbiting particles [焑].

COSY has a history of highly successful operation of cooled polarized beams and targets - in fact, COSY is a unique facility for spin physics with hadronic probes on a world-wide scale. The IKP-COSY environment, including the theoretical group is thus ideally suited for a major (mediumsized) project involving spin and storage rings as it will be required for the search for permanent EDMs of charged fundamental particles (e.g., protons, deuterons, and other light nuclei). We are planning to search for EDMs of the proton and other charged particles in a storage ring with a statistical sensitivity of $\approx 2.5 \times 10^{-29} \mathrm{e} \cdot \mathrm{cm}$ per year, pushing the limits even further and with the potential of an actual particle-EDM discovery.

The proposed new method employs radial electric fields (and magnetic fields) to steer the particle beam in the ring, magnetic or electric quadrupole magnets to form a strong focusing lattice (e.g. FODO), and internal polarimeters to probe the particle spin state as a function of storage time. An RF-cavity and sextupole magnets will be used to prolong the SCT of the beam. For protons, it requires building a storage ring with a highly uniform radial E-field with strength of $\approx 17 \mathrm{MV} / \mathrm{m}$ between stainless steel plates about $2 \mathrm{~cm}$ apart. The bending radius will be $\approx 25 \mathrm{~m}$ and including the straight sections, such a machine would have a physical radius of $\approx 30 \mathrm{~m}$. The so-called magic momentum of $0.7 \mathrm{GeV} / c(232 \mathrm{MeV})$, is the one, where the $(g-2)$ precession frequency is zero (see Table D).

Table 2: Parameters for the transverse electric and magnetic fields required to freeze the spin in an EDM storage ring of radius $r=30 \mathrm{~m}$.

\begin{tabular}{c|c|c|c} 
Particle & $p(\mathrm{GeV} / c)$ & $E(\mathrm{MV} / \mathrm{m})$ & $B(\mathrm{~T})$ \\
\hline Proton & 0.701 & 16.8 & 0 \\
Deuteron & 1.000 & -4.03 & 0.16 \\
${ }^{3} \mathrm{He}$ & 1.285 & 17.0 & -0.051 \\
\hline
\end{tabular}

\section{Precursor experiments at COSY}

The above cited tentative upper bound for the proton EDM as part of a nucleus in an electrically neutral atom, $\left|d_{p}\right|<7.9 \times 10^{-25} \mathrm{e} \mathrm{cm}$, derives from the theoretical reinterpretation of the upper bound for the EDM of ${ }^{199} \mathrm{Hg}[\mathbb{W}]$. We briefly review possible first direct measurements of an upper limit for the proton and deuteron EDM using a normal magnetic storage ring like COSY. Such experiments are widely considered must-do experiments, before embarking on the development and construction of storage rings with electrostatic deflectors.

\subsection{RFE spin rotator with Siberian snake}

Making use of a Siberian snake in COSY yields a stable longitudinal spin-closed orbit in a target section opposite the snake (see Fig. [, top panel). Using two RF E-field systems in front and behind the snake (middle and bottom panels) allows one to provide a certain degree of depolarization in the beam due to the torque $\vec{d} \times \vec{E}$, where $d$ denotes the proton electric dipole 
moment. When the RF E-field is reversed in polarity turn by turn, this torque produces a small mismatch between the two stable spin axes, hence the beam depolarizes. While the angle is ex-

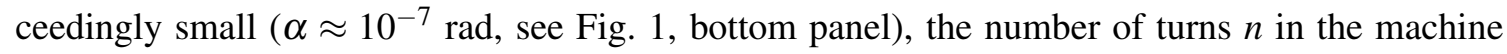
can be made very large $\left(n \approx 5 \cdot 10^{10}\right)$. The sensitivity of this approach is rather limited to values of $d \approx 10^{-17}-10^{-18} \mathrm{e} \mathrm{cm}$, but a measurement would nevertheless constitute a first direct measurement of an upper limit for the proton EDM.

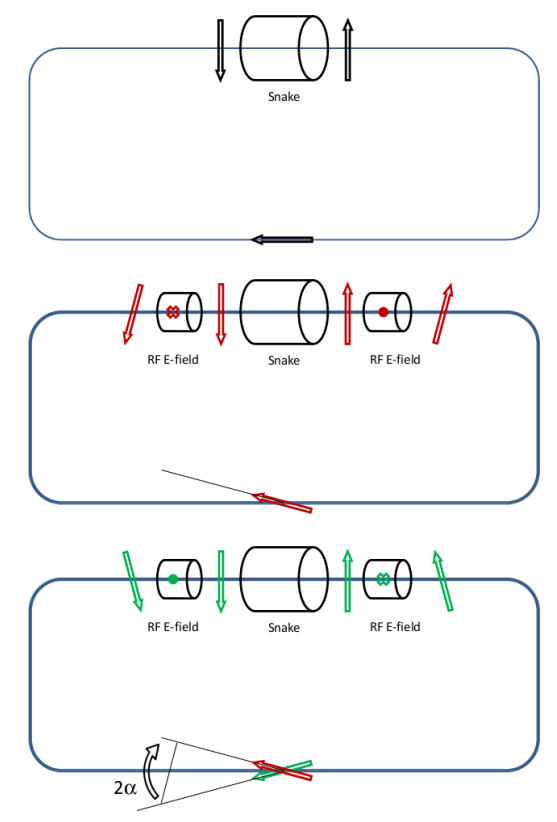

Figure 1: Concept of a proton EDM measurement using a Siberian snake in COSY. Top panel: Using the snake, the spin closed orbit is aligned along the direction of motion of the proton beam in the straight section opposite the snake. Middle panel: For odd turns in the machine, an electric RF E-field perpendicular to the ring plane in front and behind the snake rotates the stable spin axis by a small angle $\alpha$ away from the longitudinal direction. Bottom panel: For even turns in the machine where the RF E-field is reversed, the spin closed orbit is then rotated by an angle $2 \alpha$, leading after $n$ turns to a depolarization of the beam, $P(n)=P_{0} \cdot \cos (2 \alpha)^{n}$.

\subsection{Dual beam method: protons and deuterons stored simultaneously}

The dual beam method is equivalent to the $g-2$ measurement of the muon $\operatorname{EDM} d_{\mu}$, reported in [12]. It seems possible to store protons and deuterons in COSY simultaneously. The way this would be achieved is by first injecting deuterons from the injector cyclotron into COSY and accelerating them to highest energy, where the beam lifetime reaches hundreds of hours. During the deuteron storage time, the injector cyclotron is tuned for protons, the stored deuterons in COSY are decelerated to the injection momentum of $\approx 300 \mathrm{MeV} / \mathrm{c}$, electron cooled, and protons are injected. In order to cool both beams, the electron cooler voltage is switched to match the velocities of protons and deuterons for short time periods of about $10 \mathrm{~s}$.

The measurement of the muon EDM made use of the fact that the magnetic fields in the $g-2$ experiment were well known, and one was able to relate the observed additional amount of spin rotation to the muon EDM. In our scenario, we would compare the spin precession due to the deuteron EDM using the protons as a means to determine the magnetic properties of the machine. Experimentally, the task boils down to the determination of the invariant spin axes of the simultaneously stored protons and deuterons using a polarimeter. Here we envision using a polarized gas target and a detector system capable to identify $p p$ and $p d$ elastic scattering, and thereby determining all three components of the beam polarizations $\vec{P}=\left(P_{x}, P_{y}, P_{z}\right)$. Assuming a value for the proton EDM, derived from the measurement on ${ }^{199} \mathrm{Hg}$ (see Table $\mathbb{W}$ ), any mismatch of the invariant spin axes for 
deuterons and protons would be associated to an upper limit deuteron EDM. The sensitivity of this method to $d_{d}$ would be similar to the one achieved in the $g-2$ determination of $d_{\mu}$, i.e., amount to about $d_{d}=10^{-19} e \cdot \mathrm{cm}$.

\subsection{Morse-Orlov-Semertzidis resonance method for EDM measurements in storage rings}

This idea for a measurement using an all magnetic ring is described in [[13]]. One would inject sideways polarization into a machine with a vertical invariant spin axis, the EDM produces a growing vertical polarization $P_{y}$, and using two sub-beams with different machine tunes that would be independently modulated, allows one to isolate the EDM of the orbiting particles. The sensitivity of this method for protons is estimated to reach $d_{p}=10^{-29} \mathrm{e} \cdot \mathrm{cm} / \mathrm{yr}$, but because of systematic errors, the idea is presently no longer pursued at BNL. For COSY, in terms of precursor EDM measurements, this idea is being considered, although detailed evaluations have not been looked into yet.

\subsection{The resonance EDM effect with RFE flipper}

This is our favorite option and here we unfold its features in some more detail. The idea is to supplement COSY with an RFE flipper which runs at a frequency tuned to the spin tune $G \gamma$.

A non-vanishing EDM, $\vec{d}=e d \vec{S}$, gives rise to a precession of the spin $\vec{S}$ in an electric field $\vec{E}$ with $\omega_{\mathrm{EDM}}=e d E$. A single pass through the flipper of length $L$ with a radial electric field $\vec{E}$ would tilt the initial vertical spin $\vec{S} \| S_{y}$, and generate a longitudinal component $S_{z}=S_{y} \cdot \alpha$, where $\alpha=d E L / \beta c$. To appreciate the complexity of the task, for a beam of deuterons with $T=100 \mathrm{MeV}$, a RFE flipper of length $L=1 \mathrm{~m}$, a realistic electric field of $E=15 \mathrm{kV} / \mathrm{cm}$, and $d=10^{-23} \mathrm{~cm}$, one finds $\alpha=2.4 \cdot 10^{-12}$.

\subsubsection{The coherent buildup of the EDM effect: single spin problem}

The so generated longitudinal spin would precess in the magnetic field of the ring by an angle $\theta_{o}=2 \pi \gamma G$ per revolution, where $G$ is the anomalous magnetic moment. For the deuterons at injection energy, $\theta_{o} \approx-0.15$. Compared to the ring circumference, such a flipper can be treated as a point-like element. One could readily model the polarization evolution by the $\mathrm{SO}(3)$ spin rotation matrices applied to a classical polarization vector; in view of the minuscule $\alpha$ the change of the magnitude of the in plane polarization, $S_{\|}=\left(S_{x}^{2}+S_{z}^{2}\right)^{1 / 2}$, per pass is well approximated by $S_{\|}(i+1)=S_{\|}(i)+S_{y} \alpha \cos \theta(i)$. Upon summing over $k$ passes, one obtains

$$
S_{\|}=S_{y} \sum_{i=1}^{k} \alpha \cos \left(i \theta_{0}\right)
$$

which for a static electric field, $\alpha=$ const, would simply oscillate around zero. Evidently, the electric field of the flipper must by modulated in sync with the precession of the spin: $E=E_{0} \cos \left(i \theta_{0}\right)=$ $E_{0} \cos \left(\theta_{0} v t\right)$, i.e., $\alpha=\alpha_{0} \cos \left(\theta_{0} v t\right)$, where $v$ is the ring frequency, resulting in the Master Formula

$$
S_{\|}(t)=S_{y} \sum_{i=1}^{k} \alpha_{0} \cos ^{2}\left(i \theta_{0}\right)=\frac{1}{2} S_{y} \alpha_{0} v t .
$$

Only the resonance condition furnishes the coherent build-up of the EDM signal. Swapping the harmonic modulation for the rectangular one would enhance the EDM signal by the factor $4 / \pi$ : 


$$
S_{\|}(t)=S_{y} \sum_{i=1}^{k} \alpha_{0}\left|\cos \left(i \theta_{0}\right)\right|=\frac{2}{\pi} S_{y} \alpha_{0} v t .
$$

For the sake of analytic simplicity, we focus on the harmonic RFE flipper, which must run at a frequency $v_{F}=\gamma G v$, and by a judicious choice of the deuteron energy one could readily stay away of depolarizing resonances in the machine.

\subsubsection{The RFE flipper disturbs the orbit and the spin tune}

The standard spin tune $\gamma G$ only holds for a pure magnetic ring, when the spin precesses in precisely the same magnetic field that also bends the particle momentum. The RFE flipper would generate an oscillating radial momentum $\Delta p_{r}=e E_{0} L \cos \left(G \gamma v t_{i}\right) / \beta c$ per $i$-th pass, which is offtune with the ring frequency. For the above specified RFE filter and $100 \mathrm{MeV}$ deuterons the bending angle is about $\pm 2 \cdot 10^{-3}$, well within the machine acceptance of COSY. The bending can be viewed as generated by an equivalent in-phase oscillating magnetic field $B_{F}=E / \beta$ which does not contribute to the spin tune, though. The net effect is a frequency modulation of the spin tune, $G \gamma \rightarrow G \gamma\left[1-z_{0} \cos \left(i \theta_{0}\right)\right]$ where $z_{o} \ll 1$ is $B_{F, 0} L$ in units of the ring $\int B d z$. Then our Master Formula entails a negligible impact of this frequency modulation of the spin tune:

$$
\begin{array}{r}
S_{\|}(t)=S_{y} \sum_{i=1}^{k} \alpha_{0} \cos \left(i \theta_{0}\right) \cos \left(i \theta_{0}\left[1-z_{0} \cos \left(i \theta_{0}\right)\right]\right)= \\
S_{y} \sum_{i=1}^{k} \alpha_{0} \cos ^{2}\left(i \theta _ { 0 } \left[\left(1-\frac{1}{2} z_{0}^{2} \cos ^{2}\left(i \theta_{0}\right)\right]=\frac{1}{2} S_{y} \alpha_{0} v t\left(1-\frac{3}{8} z_{0}^{2}\right) .\right.\right.
\end{array}
$$

\subsubsection{Polarimetry and bunched vs. coasting beam}

Each individual particle for the first time enters the RFE flipper with a certain $\vec{S}_{y}(0)$, which remains stable, and a certain in-plane component $\vec{S}_{x z}(0)$. Upon $k$ revolutions, the overall polarization vector can be decomposed as $\vec{S}(t)=\vec{S}_{y}(0)+\hat{R}_{y}(k) \vec{S}_{x z}(0)+\vec{S}_{\|}(t)$, where $\hat{R}_{y}(k)$ is a matrix of spin rotation around the $y$-axis upon $k$ revolutions and $\vec{S}_{\|}(t)$ is the in-plane polarization generated by the RFE flipper. Upon averaging over an ensemble $\left\langle\vec{S}_{x z}(0)\right\rangle=0$, we keep $\vec{S}_{y}(0)$ for $\left\langle\vec{S}_{y}(0)\right\rangle$. For a finite-length bunch and/or coasting beam our earlier derivation holds for a particle which enters the flipper at $t=0$. Particles which are behind by a fraction $0<z<1$ of the ring circumference enter the flipper at a different field advanced by time $\Delta t=z / v$ and the modified Master Formula reads

$$
S_{\|}(z, t)=S_{y} \alpha_{0} \sum_{i=1}^{k} \cos \left(i \theta_{0}\right) \cos \left(i \theta_{0}+z \theta_{0}\right)=\frac{1}{2} S_{y} \alpha_{0} v t \cos \left(z \theta_{0}\right)
$$

The bunch is point-like and its polarization is uniform if the length of the bunch $z_{b}$ satisfies the condition $z_{b} \theta_{0} \ll 1$.

The longitudinal component $S_{z}$, and the radial one $S_{x}$ would oscillate, leading to $S_{z}=S_{\|}(t) \cos \left(\theta_{0} v t\right)$ and $S_{x}=S_{\|}(t) \sin \left(\theta_{0} v t\right)$, as shown in Fig. $\square$, where we show the results of modeling with spin rotation matrices. One would readily extract $S_{\|}(t)$ from the relevant Fourier component of the up-down asymmetry

$$
A_{u / d}=\frac{\int d t\left[N_{u p}(t)-N_{d o w n}(t)\right] \sin \left(\theta_{O} v t\right)}{\int d t\left[N_{u p}(t)+N_{d o w n}(t)\right]} \propto A_{N} S_{\|}(t),
$$



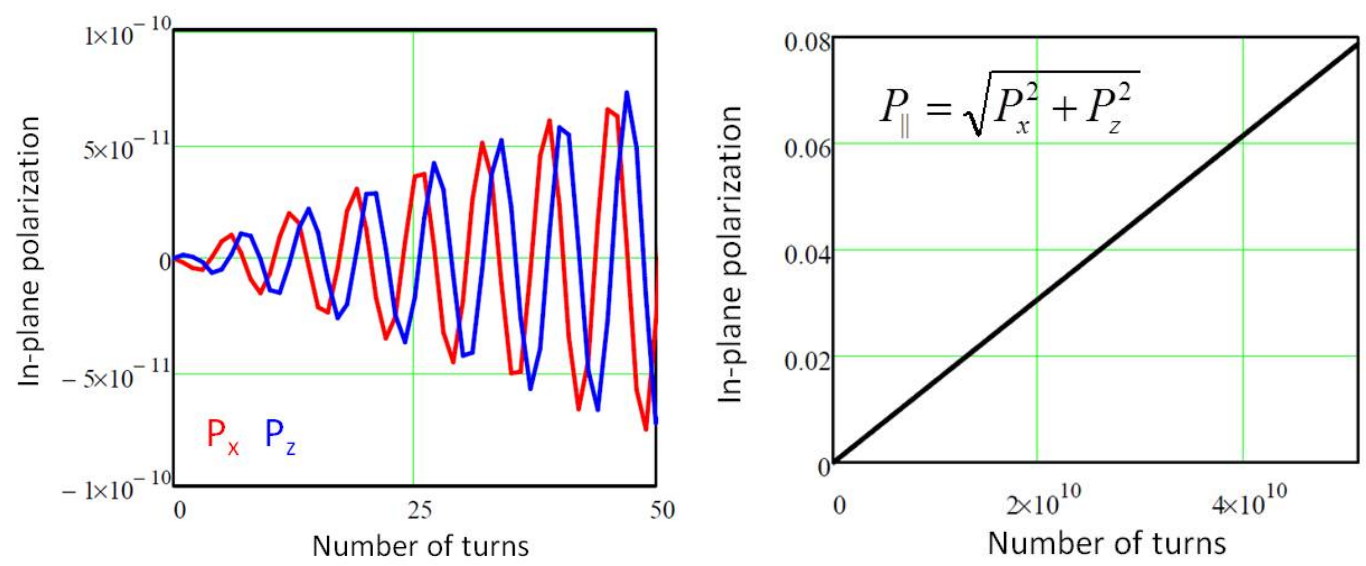

Figure 2: Left panel: Oscillating in-plane beam polarization components $P_{x}$ and $P_{z}\left(S_{x}\right.$ and $\left.S_{z}\right)$ for the first 50 turns (revolutions) in the machine. Right panel: Evolution of the magnitude of the in-plane polarization $\left.P_{\|}=\sqrt{(} P_{x}^{2}+P_{z}^{2}\right)$ during a spin coherence time of $10^{5} \mathrm{~s}$, which, under the specified conditions using 100 $\mathrm{MeV}$ deuterons in COSY corresponds to a total of $5 \times 10^{10}$ turns in COSY.

where $N_{\text {up } / \text { down }}(t)$ are the corresponding count rates - this is a familiar technique. A simultaneous measurement of both $S_{x}$ and $S_{z}$ would have been an important cross check, but a word of caution is in order: the in-plane magnetic fields are forbidden as the ordinary magnetic moment would cause the false precession of the vertical-to-in-plane spin. This seems to preclude the $S_{z}$ polarimetry on a longitudinally polarized internal target with longitudinal holding magnetic field.

The use of a transversely polarized internal target, however, seems possible, although during the spin-flip process, it must run with empty cell but vertical guide field switched on, one would inject polarized particles into the cell only at the polarimetry stage, and, since one does not want to change the holding field polarity during the measurement, injection of different states from the source is necessary.

\subsubsection{Diffusion of the in-plane spin and spin coherence time}

The extremely small single-pass rotation $\alpha_{0}$ in the RFE flipper can only be overcome by an extremely large number of turns $v t$. The in-plane spins accumulated during the flipper process must all rotate coherently at one and the same rotation angle $\theta=\theta_{0} v t$. There is an important distinction between the lifetime of the polarization along the stable-spin axis, the spin coherence of the in-plane polarization when the beam idly rotates in the storage ring and the spin coherence during the build-up of the in-plane polarization. Our interest here is in the latter.

To a first approximation $S_{y}$ is preserved irrespective of what happens to the rotating in-plane component of the spin. The spin tune $\theta_{0}=\gamma_{0} G$ varies from revolution to revolution and from stored particle to particle because of the momentum fluctuations, $\theta=\theta_{0}+2 \pi G \delta \gamma=\theta_{0}+\delta \theta$. Here $\gamma_{0}$ is defined for the average beam momentum, ensured by cooling the beam, and by the very definition $\langle\delta \gamma\rangle=0$. The RFE flipper is run at fixed frequency. Correspondingly, the Master Formula for $S_{\|}$ 
is modified to

$$
S_{\|}=S_{y} \alpha_{0} \sum_{i=1}^{k} \cos \left(i \theta_{0}+\Delta_{i}\right) \cos \left(i \theta_{0}\right)
$$

where $\Delta_{i}=\sum_{n=1}^{i} \delta \theta_{n}$ is a cumulant mismatch of the in-plane spin rotation before the $i$-th pass through the RFE flipper. The terms linear in $\Delta_{i}$ vanish in the Taylor expansion, $\left\langle\Delta_{i}\right\rangle=0$, while $\left\langle\Delta_{i}^{2}\right\rangle=i(2 \pi)^{2} G^{2}\left\langle\delta \gamma^{2}\right\rangle$, and

$$
S_{\|}=S_{y} \alpha_{0} \sum_{i=1}^{k} \cos ^{2}\left(i \theta_{0}\right)\left(1-i 2 \pi^{2} G^{2}\left\langle\delta^{2} \gamma\right\rangle\right)=S_{y} \frac{1}{2} v t\left(1-\pi^{2} v t G^{2}\left\langle\delta \gamma^{2}\right\rangle\right),
$$

which describes the diffusion spread of the in-plane polarization.

Still another source of diffusion is the fluctuation of the revolution (transit) time $\tau$, described in terms of slip-factor, $\delta \tau / \tau=\eta \delta \gamma / \gamma \beta^{2}$, where

$$
\eta=\alpha_{\mathrm{MC}}-\frac{1}{\gamma^{2}}
$$

and $\alpha_{\mathrm{MC}}$ is the momentum-compaction factor [14]]. It produces a slip of the phase of the RFE flipper per pass $\delta \theta=2 \pi \gamma G \nu \delta \tau$, the Master Formula will take the form

$$
S_{\|}=S_{y} \alpha_{0} \sum_{i=1}^{k} \cos \left(i \theta_{0}+\Delta_{i}\right) \cos \left(i \theta_{0}+\eta \Delta_{i} / \beta^{2}\right)
$$

and the above spin diffusion rate acquires an extra factor

$$
\left(1-\frac{\eta}{\beta^{2}}\right)^{2}=\frac{1}{\gamma^{4} \beta^{4}}\left(1+\beta^{2} \gamma^{2}-\alpha_{\mathrm{MC}} \gamma^{2}\right)^{2}
$$

For non-relativistic particles $-\eta / \beta^{2} \approx 1 / \beta^{2}$ and the in-plane spin-diffusion is entirely dominated by the flipper phase slip.

The relevant spin coherence time (SCT) of a certain particle species equals

$$
\tau_{S C} \approx=\frac{\gamma^{2}}{\pi^{2} G^{2} v\left(1+\beta^{2} \gamma^{2}-\alpha_{\mathrm{MC}} \gamma^{2}\right)^{2}} \cdot\left\langle\left(\frac{\delta p}{p}\right)^{2}\right\rangle^{-1}
$$

Eliminating the flipper phase-slip entirely would substantially enhance the SCT to

$$
\tau_{S C}(\eta=0) \approx=\frac{1}{\pi^{2} v G^{2} \gamma^{2} \beta^{4}} \cdot\left\langle\left(\frac{\delta p}{p}\right)^{2}\right\rangle^{-1}
$$

We shall comment more on this important option below. For the reference case of $100 \mathrm{MeV}$ deuterons, $v \approx 511 \mathrm{kHz}$, and cooled beam with $\delta p / p=10^{-4}$, our estimate yields $\tau_{S C}^{d}(\eta=0) \approx$ $3 \cdot 10^{5}$, and Eq. (4.8) suggests an optimal spin flipping time of

$$
\tau_{F} \approx \frac{1}{2} \tau_{S C}
$$




\subsubsection{The proton EDM at COSY?}

An extension to the case of protons is straightforward. What really matters in the polarization buildup is a departure of $\gamma G_{p}$ form the nearest integer $\mathrm{N}: \gamma G_{p} \rightarrow \gamma \Delta G_{p}=\min \left(\gamma G_{p}-N\right)$. Indeed, $\cos \left(i 2 \pi \gamma G_{p}\right)=\cos \left(i 2 \pi\left(\gamma \Delta G_{p}+N\right)\right)=\cos \left(i 2 \pi \gamma \Delta G_{p} \theta_{o}\right)$. Correspondingly, one must run the RFE flipper at a frequency $v_{F}=\gamma \Delta G_{p}$. On the other hand, full $\gamma G_{p} v$ would enter the modulation of the polarization along the bunch, into the precession of the stored in-plane polarization, and into the above evaluation of SCT, making $\tau_{S C}^{p}=\tau_{S C}^{d} \cdot\left(\frac{G_{d}}{G_{p}}\right)^{2} \sim \tau_{S C}^{d} / 200$. It should be emphasized that purely electrostatic rings share the above spin decoherence mechanisms, although the analytic treatment would be substantially different from that for the point-like RFE flipper.

\subsubsection{Can one run an RFE flipper at higher frequency?}

Short bunches offer a possibility of operating the RFE flipper at higher frequency. As we argued above, what matters for protons is the departure of $\gamma G_{p}$ form the nearest integer $N$. Similarly, one could run the flipper at a frequency $v_{F}=\gamma G v+K v$, where $K$ is integer. Indeed, short bunches probe the $E$-field only at discrete times $t_{i}=i / v$ and $\cos \left(2 \pi i v_{F}\right)=\cos \left(i \theta_{0}+2 \pi i K\right)=\cos \left(i \theta_{0}\right)$. Evidently, for an ideal particle, the build-up of the EDM signal wouldn't depend on $K$. The limitation on the bunch length becomes much more stringent, though:

$$
z_{b}\left(\theta_{0}+2 \pi K\right)=x_{b} \theta_{0}\left(1+\frac{K}{\gamma G}\right) \ll 1 .
$$

A similar bound is imposed on the length of the flipper, $z_{F}$, in units of the ring circumference: $z_{F} \theta_{0}(1+K / \gamma G) \ll 1$.

Unfortunately, the troublesome flipper phase slip will be enhanced by the same factor $(1+$ $K / \gamma G)$. For the deuteron $K /\left|G_{d}\right| \gg 1$ and running at higher frequencies leads to an unwanted suppression of the SCT for deuterons by a factor $\left(G_{d} / K\right)^{2}$, i.e., by almost two orders in magnitude, killing the remarkable enhancement of the deuteron SCT due to its small $G_{d}$.

\subsubsection{Advantages of a rectangular (flat-top) modulated RFE flipper}

The emerging show-stopper, the phase-slip of the flipper $E$-field, can be entirely eliminated by employing a rectangular modulation of the RFE flipper,

$$
E(t)=E_{0}(-1)^{N_{F}}, \quad N_{F}=\operatorname{int}(\gamma G v t / \pi) .
$$

Indeed, the factor $(-1)^{N_{F}}$ in the Master Eq. (4.3) does not depend on the phase slip. Furthermore, with a rectangular modulation one could afford the high-frequency option. One can make sure that the $E$-field is flipped when the beam bunch is outside of the flipper, which simultaneously would eliminate the action on the bunch by magnetic fields that are generated by $d E / d t$ in the course of flipping the flipper-field. The exact rectangular modulation is not imperative, what we are asking for is a flat top when the bunch passes through the flipper. All the above arguments make the flat-top-modulated RFE flipper an extremely attractive one.

\subsubsection{In-situ studies of the systematics of EDM measurements using a magnetic flipper}

The systematics is the bane of high precision experiments. Fortunately, much of the ring systematics of the EDM precursor experiments can be measured in situ swapping the radial electric 
field in the RFE flipper for a weak radial magnetic field $B_{R}$, providing an RFB flipper, in which the magnetic moment of the deuteron will do precisely the same job as the EDM in the RFE flipper. Such studies would allow to establish experimentally the sensitivity limit well before experimenting with an RFE flipper. The corresponding magnetic spin rotation angle

$$
\alpha_{M}=\mu_{d} B_{R} L / \beta c
$$

can be made as small as necessary, eventually going down to $\alpha_{M} \sim \alpha_{0}$. This way, one would be able to check experimentally many aspects: (a) the difference between harmonic and flat-top flippers, (b) the dependence of SCT on $\delta p / p$ by heating the beam by stochastic vertical magnetic field, which would allow one to explore also a region of strong spin-diffusion, and (c) the predicted difference of SCT for protons and deuterons, etc.

\section{Summary and Conclusions}

Running the COSY ring, supplemented with the above specified flattop-modulated RFE flipper (for $100 \mathrm{MeV}$ deuterons, $v_{F}=\gamma\left|G_{d}\right| v \sim 77 \mathrm{kHz}$ ), for $\tau_{S C}^{d}=10^{5} \mathrm{~s}$ and $d_{d}=10^{-23} \mathrm{e} \cdot \mathrm{cm}$, the accumulated $C P$ violating in-plane polarization of the deuteron could be as large as $S_{\|}=0.08(0.06$ for the harmonic flipper). To reach an upper bound of $d_{d}=10^{-24} \mathrm{e} \cdot \mathrm{cm}$ polarizations of $S_{\|}=0.008$ ( 0.006 for the harmonic flipper) need to be determined, which is within the reach of state-of the-art polarimetry. Such an upper bound on the deuteron EDM of $d_{d}<10^{-24} \mathrm{e} \cdot \mathrm{cm}$ would be comparable to the results from the model-dependent reinterpretation of upper bounds on atomic EDMs [2], and sizewise close to the ballpark neutron EDM bounds. True, regarding the systematics, we have presently touched only the tip of the iceberg in a very crude analytic approach and much more scrutiny is in order. Specifically, one badly needs spin tracking tools capable of handling with controlled precision up to $\sim 10^{11}$ turns in a realistically modeled machine. With all reservations, the RFE flipper experiment at COSY looks like a promising one. We especially emphasize again here the importance of in-situ studies at COSY using very slow RF magnetic flippers to study systematic effects.

\section{Acknowledgments}

In the development of concepts for precursor experiments, outlined here, we greatly benefited from extended discussion with Bill Morse of BNL, who took on the idea of RFE flippers and suggested to us a way of operating such a device that would allow accumulation of the EDM signal during the SCT, and in particular, it is Eq. (4.2) which has been derived by him. In addition, we would like to acknowledge fruitful discussions with Rudolf Maier, Andreas Lehrach, Yuri Senichev, and Hans Ströher.

\section{References}

[1] A. Sakharov, Pisma Zh.Eksp.Teor.Fiz. 5, 32 (1967), Reprinted in *Kolb, E.W. (ed.), Turner, M.S. (ed.): The early universe* 371-373, and in *Lindley, D. (ed.) et al.: Cosmology and particle physics* 106-109, and in Sov. Phys. Usp. 34 (1991) 392-393 [Usp. Fiz. Nauk 161 (1991) No. 5 61-64]. 
[2] B. L. Roberts and W. J. Marciano (eds.), Lepton Dipole Moments, Advanced Series on Directions in High Energy Physics, Vol. 20 (World Scientific, 2010).

[3] S. Weinberg, AIP Conf.Proc. 272, 346 (1993).

[4] V. Flambaum, I. Khriplovich and O. Sushkov, Nucl.Phys. A449, p. 750 (1986).

[5] V. Flambaum, I. Khriplovich and O. Sushkov, Phys.Lett. B162, 213 (1985).

[6] Y. K. Semertzidis, AIP Conf.Proc. 1182, 730 (2009).

[7] J. H. Smith, E. M. Purcell and N. F. Ramsey, Phys. Rev. 108, 120(Oct 1957).

[8] S. K. Lamoreux and D. Golub, Lepton Dipole Moments, Advanced Series on Directions in High Energy Physics Vol. 20 (World Scientific, 2010), ch. 15, p. 583.

[9] C. Baker et al., Phys.Rev.Lett. 98, p. 149102 (2007).

[10] W. Griffith et al., Phys.Rev.Lett. 102, p. 101601 (2009).

[11] M. A. Rosenberry and T. E. Chupp, Phys. Rev. Lett. 86, 22(Jan 2001).

[12] G. W. Bennett et al., Phys. Rev. D 80, p. 052008(Sep 2009).

[13] Y. F. Orlov, W. M. Morse and Y. K. Semertzidis, Phys. Rev. Lett. 96, p. 214802(Jun 2006).

[14] A. Chao and M. Tigner (eds.), Handbook of accelerator physics and engineering (World Scientific, 1999). 